ÉGYPTE

monde arabe

\section{Égypte/Monde arabe}

4 | 2007

Figures de la santé en Égypte

\title{
Figures de la santé publique en Égypte : passé, présent, avenir. Introduction
}

Matthieu Fintz, Anne Marie Moulin et Saadia Radi

\section{(2) OpenEdition}

Édition électronique

URL : https://journals.openedition.org/ema/1731

DOI : 10.4000/ema.1731

ISSN : 2090-7273

Éditeur

CEDEJ - Centre d'études et de documentation économiques juridiques et sociales

Édition imprimée

Date de publication : 31 décembre 2007

Pagination : 13-34

ISBN : 2-6905838-43-4

ISSN : 1110-5097

Référence électronique

Matthieu Fintz, Anne Marie Moulin et Saadia Radi, «Figures de la santé publique en Égypte : passé, présent, avenir. Introduction », Égypte/Monde arabe [En ligne], 4 | 2007, mis en ligne le 08 juillet 2008, consulté le 07 juillet 2022. URL : http://journals.openedition.org/ema/1731 ; DOI : https://doi.org/ 10.4000/ema. 1731 


\title{
Anne Marie Moulin, Matthieu Fintz et Saadia Radi
}

\section{INTRODUCTION}

\begin{abstract}
Ce numéro spécial d'Égypte/Monde arabe rassemble des recherches de sciences sociales sur les questions de santé, de maladie et de médecine en Égypte, de ce qu'il est convenu d'appeler la naissance de l'État-nation sous le règne de Muhammad 'Alî pacha jusqu'à nos jours. II ne prétend pas dresser un panorama exhaustif de la situation sanitaire égyptienne contemporaine ${ }^{1}$. II vise à procurer sur la santé en Égypte des entrées variées, reflétant les travaux en cours des différents auteurs et suggérer les rebondissements d'une histoire qui est à la fois politique, sociale et scientifique.

Nous avons choisi d'articuler les contributions selon trois axes. Un premier ensemble s'intitule « Figures historiques de la santé publique. La médicalisation de l'Égypte ». Un deuxième ensemble, "Ordre et désordres de la santé », est centré sur les dynamiques qui animent le "champ miné du savoir médical » (Moulin, 2004, p. 21), un domaine à propos duquel les médias égyptiens évoquent volontiers des scandales, et qui figure les tensions et contradictions qui traversent la société égyptienne. Un troisième ensemble, "Vers une reconfiguration de la santé ", évoque les reconfigurations possibles de la santé sous les coups de boutoir de l'épidémie d'hépatite $C$ et de la menace de la grippe aviaire, dans le contexte de l'ouverture de la succession du président égyptien.
\end{abstract}

\section{FIGURES HISTORIQUES. LES PRÉMISSES DE LA MÉDICALISATION DE L'ÉGYPTE}

La science arabe dans le passé a fait une très large place à la médecine. L'ouvrage d'Ibn Ridwân, écrit au Xl ${ }^{\mathrm{e}}$ siècle, sur La prévention des maux du corps en Égypte (Dols, 1984) fait état d'une tradition scientifique ancienne, empruntant largement aux écrits d'Hippocrate et de Galien, relayés et amplifiés par Ibn Ruchd, Maimonide, Ibn Butlân et bien d'autres. Vivant dans l'Égypte fatimide,

1. Objectif d'un chapitre dans l'ouvrage collectif sur l'Égypte, actuellement en chantier au Cedej, Battesti V. et Ireton F. (dirs). 
Ibn Ridwân polémique avec Ibn Jazzâr, à Kairouan en Tunisie, prouvant la participation active des savants égyptiens aux controverses scientifiques au sein du Dar al-Islam, en dépit de ses divisions politiques et religieuses.

Cette tradition savante était avant tout tournée vers la prise en charge de la santé individuelle sous une double forme, préventive et curative. La première l'emportait d'ailleurs sur la seconde, comme le souligne Ibn Sînâ dans son Qânûn, en ce qu'il paraît plus simple de prévenir des maux que de les guérir. Cette action de prévention passe par un suivi attentif de la vie quotidienne du malade potentiel, qui concerne surtout les riches, les seuls qui aient le loisir de s'observer et de faire appel à des hommes de l'art à leur domicile. La médecine médiévale porte une particulière attention aux " choses non naturelles ", selon la terminologie de Galien, qu'elle peut influencer : le régime alimentaire, l'exercice physique et la vie sexuelle. Elle prodigue inlassablement des conseils détaillés et quelques remèdes, modulés en fonction de l'âge, de la saison et de l'activité professionnelle : c'est une médecine taillée en quelque sorte sur mesure, un idéal que notre médecine d'aujourd'hui est loin d'atteindre.

Si les épidémies ont toujours mis au défi les gouvernements en semant le désordre (Dols, 1972 et Moulin, 1996), la gestion organisée de la santé collective, promue en Occident sous le nom d'hygiène publique, se dessine vraiment en Égypte au début du XIX $x^{\mathrm{e}}$ siècle où elle constitue une des facettes de la création de l'État moderne par Muhammad 'Alî (Abbas, 2000). Le pacha, pendant son long règne de 1805 à 1849, quelles que soient par ailleurs les controverses sur les sens de son action politique ${ }^{2}$, a jeté les bases d'une administration sanitaire enregistrant la croissance de la population et lui imposant des mesures collectives comme la vaccination antivariolique (Clot, 1840). Commencée au sein de l'armée, la médicalisation de l'Égypte est indissociable de l'initiation de la modernisation sous Muhammad 'Alî.

Le texte de Panayotis Krokidas et Athanasios Gekas rapporte un épisode peu connu où, sous l'effet de la politique expansionniste du pacha, l'Égypte joue dans la région un rôle de pionnier dans le domaine de la santé publique. La scène se passe en Crête, alors sous pouvoir ottoman (jusqu'en 1898), au moment où les réformes de Muhammad 'Alî connaissent un début de réalisation local, à l'occasion des campagnes d'Ibrâhîm pacha et se présentent comme un modèle. Le refus de tenir compte des divisions religieuses et de traiter différemment dhimmî (statut légal des non-musulmans dans un État islamique) et musulmans, dès lors qu'il s'agit de santé publique et de prévention des épidémies, trouve un écho particulier en Crête où la population est en majorité composée de rayas chrétiens. Sur le sol égyptien, Muhammad 'Alî a décidé la vaccination antivariolique obligatoire pour

2. Pour un aperçu des controverses entre les historiens nationalistes qui voient dans I'action du pacha la création d'un État-nation et ceux qui décrivent l'émancipation d'un féodal ottoman, voir K Fahmi, 1997. 
tous. En dépit des protestations des consuls, le pacha s'est refusé d'exempter certaines catégories de la population. Aux consuls rappelant les privilèges des Capitulations, Muhammad 'Alî a répliqué avec force qu'en matière d'intérêt public (al-maslahat al-'amma) il ne saurait y avoir d'exception pour les étrangers. De même, dhimmî et musulmans doivent également observer les règles de quarantaine et I'obligation du vaccin. Dans cette conception de la santé publique, s'adressant à des citoyens solidaires face à la contagion, appliquée en Crête dans les années 1830, on peut voir l'ébauche d'un État de citoyens sans particularismes ethniques et religieux qu'un Mustafâ Kamâl imposera en Turquie.

Muhammad 'Alî a également tenté de renforcer les prérogatives de l'État à propos de la composition et du fonctionnement du Conseil sanitaire d'Alexandrie, qui gérait les quarantaines établies autant pour protéger le territoire que répondre aux demandes des pays d'Europe. Le Conseil était, au début de son règne, constitué de façon prédominante de médecins étrangers et de représentants consulaires des grandes puissances européennes. Dès qu'il l'a pu, le pacha s'est efforcé de prendre le contrôle du Conseil en introduisant une majorité de représentants égyptiens de I'administration, dont certains médecins fraîchement émoulus de l'École de médecine qu'il avait fondée en 1827, avec l'aide du Dr Antoine Barthélemy Clot, resté dans I'histoire sous le nom de Clot-bey. Sylvia Chiffoleau rappelle comment la lutte contre les épidémies est devenue de façon croissante au xix siècle un enjeu pour les puissances internationales désireuses de se protéger à l'égard d'un Orient considéré comme le berceau des pestilences en reportant le contrôle à ses frontières. Elle retrace les enjeux politiques et scientifiques du Conseil sanitaire d'Alexandrie, entre 1865 et 1938.

Au cours de la deuxième moitié du xix ${ }^{\mathrm{e}}$ siècle, les grandes conférences sanitaires internationales qui se succèdent se préoccupent avant tout du choléra, plus accessoirement de la peste, dans le contrôle aux frontières des voyageurs et des marchandises. Le pèlerinage de la Mecque, brassant des populations de voyageurs venant de partout pour communier dans l'unité de l'umma sur les lieux saints, est de plus en plus désigné comme un péril épidémique majeur par les observateurs occidentaux, à mesure que s'accélère la vitesse des transports. Finies les caravanes qui s'éteignaient dans le désert, frappées par la contagion, les bateaux venus du Hedjaz débarquent désormais rapidement sur la côte de la mer Rouge leur cargaison de pèlerins souvent transportés dans des conditions d'hygiène déplorables, et le choléra fait son apparition sur le sol égyptien peu de temps après. De nos jours la combinaison de la vaccination et du traitement antibiotique n'a pas réussi à éliminer totalement le choléra, et le pèlerinage fait toujours l'objet d'une surveillance épidémiologique particulière, clinique et bactériologique.

Muhammad 'Alî s'était préoccupé de la santé publique à propos des épidémies, mais celles-ci étaient loin de résumer toutes les causes de mortalité massive. En 1822, son aménagement du canal Mahmûdiyya reliant Alexandrie 
au Nil avait drainé des milliers de fellahs requis pour les travaux. Sur 350000 (Mikhail, 2007), un tiers environ était mort en deux ans de travaux, de la peste, d'accidents du travail, de malnutrition ou de mauvais traitements. Cette hécatombe a pu jouer un rôle dans la décision du pacha d'ouvrir une école de médecine quelques années plus tard.

Pendant la période khédiviale, la politique de grands travaux autour du canal de Suez, entamée en 1857, a entraîné des déplacements massifs d'ouvriers étrangers venus d'un peu partout et de fellahs égyptiens mal nourris, sur un chantier gigantesque qui a duré plus de dix ans. La mortalité a été élevée, en raison des accidents du travail et des épidémies de choléra liées au manque d'eau potable et l'absence d'hygiène fécale, et aussi du paludisme : I'installation de ces masses d'hommes a favorisé la multiplication de flaques d'eau stagnante et les moustiques ont pullulé dans une région jusque là désertique et peu peuplée ${ }^{3}$. La mortalité est restée néanmoins très inférieure à celle du canal Mahmûdiyya : autour de $8 \%$. On peut spéculer sur les différents facteurs à l'origine de cette amélioration relative : le rôle d'une poignée de médecins de zèle et de compétence inégales, la part d'une meilleure organisation sociale et technique du travail... Les premiers accords de Lesseps avec Saïd pacha comportaient la clause : "Les ouvriers auront droit à la nourriture, au logement et aux services médicaux et d'hygiène »(Beatty, 1957, p. 128). Comme Céline Frémaux le rapporte, la Compagnie du canal de Suez a prétendu jeter les bases d'un habitat sanitaire moderne. Elle décrit les projets d'un urbanisme nouveau à Ismaïlia sur le Canal, supposé fournir un modèle pour les villes à naître, à l'occasion de l'industrialisation progressive du pays : habitat en dur avec séparation stricte des animaux domestiques, systèmes de distribution d'eau et de drainage et de voirie. Pourtant, de cette période de la construction du canal de Suez, plus que la modernisation de l'habitat, les manuels d'histoire en usage dans la République arabe unie retiennent surtout le coût humain, qu'il faudrait comparer aux pertes du canal de Panama ou de la construction des chemins de fer en Afrique et en Asie et même en Europe (tunnels, par exemple).

Après I'Indépendance, I'État nassérien, négligeant la parenthèse britannique voire celle de la monarchie constitutionnelle, s'est posé comme héritier direct de l'état fondé par Muhammad 'Alî, tout en revendiquant l'idéal, inspiré par le socialisme, d'un État-Providence destiné à combler les aspirations des masses à une meilleure santé autant qu'à camper une grande nation sur l'échiquier du monde. Pendant cette période éprise de progrès technique et à l'époque du bond en avant de la construction du barrage d'Assouan, la santé publique a été l'objet des attentions du régime qui entendait la promouvoir au niveau de ses ambitions générales. C'est l'époque, après une longue stagnation de la démographie médicale, de la multiplication des praticiens (Chiffoleau, 1997) et des facultés de médecine, de la construction de nouveaux hôpitaux,

3. On trouvera une bonne description dans Erckmann-Chatrian, 1963. 
y compris dans les villes moyennes, et malgré un décalage persistant entre le Caire et le reste du pays et entre le Delta et la Haute-Égypte.

L'ouvrage d'Ahmad Amîn, que présente ici Emmanuelle Perrin, est précisément publié en 1953, moment charnière de la médicalisation de l'Égypte, entre les ambitions réformistes dont les médecins égyptiens se font l'écho dès les années 1930 (Gallagher, 1990) et la révolution des Officiers libres. L'hygiène rurale devient d'actualité ${ }^{4}$ et les élites se préoccupent des conditions de vie des populations paysannes (cf. Ayrout, 1938 ; Blackman, 1948). L'ouvrage fait une large place aux pratiques populaires de santé. II est tout à fait caractéristique d'un souci de recueillir un patrimoine folklorique mais aussi de la mentalité des intellectuels de la période nassérienne, quelque peu sourcilleux vis-à-vis de ces mêmes coutumes populaires. L'inventaire de la « médecine des vieilles femmes » ou de la quenouille (tibb al-ruqâ) reste aujourd'hui un témoignage très précieux sur des croyances ou des rites qui ont subsisté en partie seulement jusqu'à aujourd'hui. Ahmad Amîn I'a rédigé avec minutie et parfois attendrissement, mais sur le ton d'un instituteur qui bien loin de magnifier une tradition culturelle pétrie de références religieuses, vise à réformer une mentalité primitive dont il espère qu'elle s'amendera avec les progrès de l'instruction pour tous, un des secteurs clé du programme nassérien.

\section{ORDRE ET DÉSORDRES : LA SANTÉ DANS L'ÉGYPTE CONTEMPORAINE}

En survolant la démographie égyptienne du xıx siècle à nos jours, le bilan semble très positif. La population égyptienne, forte de deux à trois millions au début du xIx ${ }^{\mathrm{e}}$ siècle aurait triplé cent ans plus tard, et elle atteint aujourd'hui plus de 70 millions d'habitants. Les données statistiques provenant du CAPMAS (Moriconi-Ebrard et Bayoumi, 2005), reposant sur un siècle de recensements, enregistrent une amélioration spectaculaire de l'espérance de vie à partir de la deuxième moitié du xix ${ }^{\mathrm{e}}$ siècle. Sans atteindre le niveau des pays les plus favorisés, l'Égypte a maintenant une espérance de vie qui dépasse 65 ans pour les hommes et atteint 70 ans pour les femmes (Courbage, 1994 ; Al Zanaty, et Way, 2000). Au point que sans connaître les affres d'un Japon submergé par ses centenaires, l'Égypte commence elle aussi à héberger un surplus de personnes âgées ${ }^{5}$. Ceci risque à terme de relativiser la baraka que la tradition

4. On peut se souvenir du rapport ambigu, entre rêve et réalité, que Hasan Fathî, fils d'un propriétaire terrien du Delta et futur architecte de la réhabilitation de l'habitat rural en Égypte dans les années 1950, écrit avoir entretenu pendant sa jeunesse avec la campagne, " a paradise darkened from above by clouds of flies, and whose streams flowing underfoot had become muddy and infested with bilharzia and dysentery ", Fathy H., 2004, p 2.

5. Voir bibliographie détaillée dans Boggatz et Dasson, 2005. 
attribuait à la présence d'un centenaire dans un quartier et de faire perdre aux " anciens » les privilèges dont ils jouissent dans les quartiers urbains comme ils en jouissaient au village.

L'Égypte aujourd'hui traverse ce qu'on appelle couramment la « transition épidémiologique $»^{6}$. Elle passe $d^{\prime}$ un régime dominé par les maladies infectieuses et une forte mortalité maternelle et infantile à un régime marqué par les maladies chroniques, cancéreuses et cardio-vasculaires. Elle est aussi passée d'un régime de catastrophes collectives à une prédominance de maladies individuelles comme les maladies métaboliques, au premier rang le diabète et les affections liées à la surcharge pondérale. L'obésité est un nouveau fléau, lié dans un pays qui reste marqué par la pauvreté à une "malbouffe » très moderniste (grignotage de « junk food » et de sucreries, en particulier chez les enfants) et qui dispose de peu d'équipements sportifs populaires.

Le bilan démographique très positif est-il lié fortement à la médicalisation? Ivan Illich I'avait jadis contesté dans un livre célèbre (Illich, 1975). On peut légitimement reposer la même question en ce qui concerne l'Égypte, où les progrès de la médicalisation ne font pas de doute.

Dès 1827, avec la réorganisation de l'hôpital d'Abû Za'bal dans la banlieue du Caire et la création de celui de Qasr al-'Aiynî en 1831, aujourd'hui hôpital de I'université du Caire, l'Égypte avait repris le flambeau d'une tradition médiévale, celle des fondations hospitalières. L'Orient a souvent revendiqué I'invention de l'hôpital non point seulement comme lieu d'hébergement et de secours mais comme lieu d'enseignement clinique. Ne rentrons pas dans la controverse de priorité (Dols, 1987) entre Orient et Occident, pour dire simplement que l'hôpital a constitué, là comme ailleurs, pendant longtemps plutôt un repoussoir pour les riches qui préféraient se faire soigner à domicile. En Europe, I'hôpital moderne qui sert à la démonstration des maladies pour l'édification des étudiants n'est vraiment devenu le lieu d'introduction et d'expérimentation des innovations scientifiques qu'à la fin du XIX siècle. $\mathrm{C}^{\prime}$ est alors que se sont multipliés les actes chirurgicaux dans des conditions d'asepsie et d'anesthésie et que des " cures miracle " liées aux recherches de laboratoire, comme la sérothérapie antidiphtérique, la chimiothérapie, les antibiotiques etc. ont fait leur apparition, entraînant l'entrée de toutes les classes de la société dans les services hospitaliers. Ce type d'hôpital est bien implanté dans l'Égypte contemporaine.

Dans ce pays, I'ancienneté de la médecine arabe et de sa tradition savante, largement commune avec la médecine occidentale, a concouru à un préjugé favorable à la biomédecine largement répandu dans la population. Elle explique aussi l'absence d'individualisation d'une tradition autonome opposable à la médecine occidentale, comme on a pu I'observer en Inde, en Chine, au ViêtNam et dans d'autres pays. L'émergence, tardive par rapport à l'indépendance

6. Identifiée comme telle par Omran, 1971. 
du pays, d'une médecine à coloration islamique, n'a pas constitué une véritable alternative, avec des enseignements et des filières autonomes. L'insertion d'une faculté de médecine dans l'enceinte de l'université Al-Azhar, souhaitée par plusieurs réformateurs entre les deux guerres dont le cheikh d'Al-Azhar, alMarâghî (Costet-Tardieu, 2005), s'est réalisée finalement sous Sadate. Elle n'a pas eu pour conséquence une relecture radicale des sciences fondamentales ou de la clinique. Ce n'est que récemment que l'apprentissage de la dissection et l'examen du corps des malades à travers la barrière des sexes ont été rediscutés par certaines instances universitaires religieuses. Mais comme on peut le voir dans la pratique des dispensaires de quartier gérés par les Frères musulmans ou des associations islamistes comme al-Jamâ'a al-Islâmiyya, le dilemme moral posé par les procédures de la médecine est souvent résolu en fournissant un « cadre » islamique garant de la respectabilité et de la moralité des professionnels : si le "style » peut différer, les pratiques s'avèrent finalement assez proches dans les hôpitaux publics et les hôpitaux et dispensaires confessionnels de différentes obédiences (Turc, s. d.).

L'Égypte illustre l'établissement du pouvoir médical (cf. Léonard, 1981 et Goubert, 1982), I'accès des médecins à des positions de notabilités, et leur présence dans les cercles dirigeants; et la médicalisation de la société, qui implique leur intervention dans de multiples domaines, de l'école à l'usine et au tribunal et leur position d'experts bien au delà des limites des lieux médicaux proprement dits. Au début du xxl ${ }^{\mathrm{e}}$ siècle, avec le nombre élevé de ses hôpitaux et de médecins, tout conflue donc pour présenter au citoyen égyptien l'image favorable d'une médecine scientifique puissante et efficace par les interventions qu'elle autorise et les médicaments qu'elle dispense.

Le public égyptien ne rejette pas, loin de là, la biomédecine avec laquelle il s'est familiarisé depuis l'époque lointaine de Muhammad 'Alî. Pourtant la représentation qu'il se fait de ses services est loin d'être positive. En 2005, une grande enquête d'opinion a été réalisée par Internet, par téléphone et par la poste, auprès du grand public pour recueillir les avis sur le système de santé et le fonctionnement de l'assurance santé existante et a révélé une grande insatisfaction, on peut même dire une grande frustration par rapport aux attentes ${ }^{7}$. Les chapitres qui suivent suggèrent un contraste parfois violent entre les espérances placées en une médecine moderne dont les hommes espèrent, comme ailleurs, qu'elle atténuera ou résoudra une partie de leurs maux, et la réalité quotidienne. Le prestige de la médecine est incontestable, une véritable aura entoure certaines figures de proue qui apparaissent souvent dans les médias. Mais cette reconnaissance sociale va de pair avec une critique vive et même décapante qui s'exprime dans la presse, même officielle, et les conversations du tout venant. La déception marquée à l'égard du système de santé et de ses protagonistes est à la mesure de l'attente.

7. F. Clément, dans ce numéro. 
L'histoire de I'hépatite C donne la mesure de cette déconvenue. L'hépatite C est un fléau qui frappe toutes les classes sociales, en particulier les professions de santé qui lui paient un lourd tribut. L'Égypte a le triste privilège de compter parmi les pays au monde ayant la plus haute prévalence ; dans la génération actuellement entre trente et cinquante ans, le taux d'hépatites chroniques peut atteindre $30 \%$. L'ampleur du désastre a été révélée par un médecin égyptien travaillant dans un centre de maladies du foie, le Dr Yâsîn 'Abd al-Ghaffâr, en 1995, et confirmée par des tests systématiques exigés par les pays du Golfe chez les travailleurs migrants avant leur entrée sur le territoire (Radi, 2006).

Cette histoire est marquée par une blessure intime nationale. L'épidémie égyptienne est particulière en ce qu'elle a été favorisée par un programme de santé publique contre la bilharziose, poursuivi jusqu'au début des années 1980 : il s'agissait d'un traitement de masse systématique (c'est-à-dire sans dépistage préalable de la maladie) par injection d'un médicament, le tartrate d'émétine. L'insuffisance de stérilisation soigneuse du matériel a probablement été à l'origine d'une contamination massive de la population par le virus de I'hépatite C (Frank, Mohamed et al., 2000). Celle-ci a ainsi échangé en quelque sorte un fléau tropical ancien, connu depuis les Pharaons, la bilharziose (Farley, 1991), contre une maladie virale " émergente » résolument moderne (le virus a été identifié au laboratoire en 1989). La propagation de la maladie, à partir d'un noyau initial de personnes atteintes (environ $20 \%$ des cas) a probablement été facilitée par la lenteur des réactions du gouvernement, après la découverte des premiers cas. L'État, en dépit de quelques interpellations au Parlement, a répugné à assumer des responsabilités I'obligeant à faire face à des revendications rappelant les affaires du sang contaminé, qui en Europe ont donné lieu à des dédommagements officiels. De surcroît, les mécanismes de dissémination de l'infection n'ont pas été analysés avec précision, et peu de mesures ont été prises pour limiter la transmission, en particulier par les instruments utilisés dans les hôpitaux et par les injections, ce qui explique que les professionnels de santé soient actuellement particulièrement touchés. L'épidémie se poursuit aujourd'hui à bas bruit, et ses mécanismes de propagation ne sont pas connus avec précision. Il existe par exemple un certain nombre de cas familiaux, qui peuvent s'expliquer tout autant par des facteurs génétiques, sensibilité commune au virus, que par des facteurs environnementaux partagés dans une maisonnée.

Il existe dans les pays nantis des traitements d'une efficacité relative, mais très coûteux et nécessitant un suivi biologique. En Égypte, des protocoles adaptés aux faibles ressources locales sont aujourd'hui à l'étude. La population ne renonce pas pour autant à l'espoir d'une cure. Comme le souligne Saadia Radi, il ne s'agit pas de refus de la médecine occidentale mais de l'impossibilité matérielle d'en tirer parti. Le pluralisme médical est en grande partie une solution par défaut. Les Égyptiens empruntent toutes sortes d'itinéraires thérapeutiques, qui les amènent à revisiter les «cultures médicales » disponibles dans leur pays. Ce que l'on ne peut soigner par le médicament peut 
parfois être soigné par l'invocation d'un saint, par la Médecine du Prophète ou par une forme de médecine alternative. Chacune de ces possibilités renvoie à un imaginaire et à un passé riches où un orientaliste pourrait être tenté de retrouver la force de vieilles croyances ou l'influence toujours déterminante de l'Islam. Pourtant, si l'on y regarde de près, ces différents recours visent tout simplement à résoudre les problèmes de la vie quotidienne et alléger les souffrances, dans le contexte d'une offre de soins à la fois plurielle et inégalement répartie. Certains privilégiés ont recours à des médicaments importés d'Europe. $\mathrm{D}^{\prime}$ autres tentent des remèdes proposés par des praticiens locaux auxquels le gouvernement s'associe parfois, qui souhaiterait évidemment contribuer au développement d'une industrie pharmaceutique proprement égyptienne.

La santé fait partie des domaines instables à propos desquels la presse évoque souvent des débordements, des "scandales». Il y a eu le scandale de la mauvaise gestion des déchets médicaux, des «poches » de sang non conformes qui auraient un effet cancérigène, de la contamination de l'alimentation par les engrais et les pesticides, etc. Le dossier technique est rarement présenté en détail. La faiblesse de la communication scientifique officielle autorise toutes les rumeurs. Comme l'a démontré Véronique Campion-Vincent (Campion-Vincent, 1996), leur importance est un bon indice de dysfonctionnement de la communication.

Les rumeurs témoignent aussi d'une défiance à l'égard des médecins, dont les pouvoirs et les savoirs ont pourtant connu un essor sans précédent. On retrouve en Égypte la même situation qu'en Occident, à ceci près qu'en Europe le malentendu entre patients et praticiens se marque par un nombre croissant de procès ou ce qu'on appelle la judiciarisation de la médecine, et la revendication croissante de l'autonomie et de l'information exhaustive donnée au malade, tandis qu'en Égypte, le malaise se manifeste plutôt sous forme de « bruits » divers dont les journaux se font l'écho, sans vérifier toujours leur authenticité, laissant l'opinion encore plus perplexe sur la conduite à tenir. Le mécontentement couve mais favorise plutôt la recherche pragmatique de solutions individuelles que la revendication collective. II n'existe pas encore d'association officielle de patients vivant avec telle ou telle maladie et jouant un rôle de lobby pour actionner les politiques et stimuler la recherche.

La médecine islamique, comme nous l'avons dit plus haut, ne fournit pas la promesse $d^{\prime}$ une autre science sous les auspices de postulats nouveaux, comme des déclarations, il y a vingt ans, dans certains pays musulmans, avaient donné à le penser (Moulin, 1991). À cette époque, avait été évoquée la possibilité d'une science authentiquement islamique (Sardar, 1984) - on parlait ailleurs de «Science for the people » ou de science prolétarienne - reposant sur des postulats en rupture avec la science courante, comme le respect de l'équilibre et de la beauté de la nature créée - orientation écologique - et la justice distributive entre les membres de la communauté - orientation sociale.

En fait, comme les travaux récents des anthropologues le montrent bien, I'insatisfaction profonde des populations provient non tant des défauts ou des 
incohérences de la science, encore qu'ils soient bien réels, mais de la façon dont le système de soins moderne fonctionne concrètement. Nous ne disposons que d'études partielles sur ce sujet dans la société égyptienne, mais il est clair que comme ailleurs (Jaffré et Sardan, 2004), les prestations du système de santé au quotidien sont la source de nombreux mécontentements. La corruption existe, le capital social est toujours bienvenu pour autoriser un coupe-file, recevoir un meilleur accueil ou obtenir des médicaments dans les établissements publics où les ruptures de stocks sont fréquentes. De toute façon, la mauvaise image de ces mêmes établissements amène les usagers à privilégier des remèdes plus chers acquis dans le privé. Si l'Égypte ignore les pharmacies par terre de nombreux pays en développement, où se bradent des médicaments périmés ou même factices, la pharmacie régulière y joue un rôle vicariant de la médecine et délivre des médicaments de façon souvent excessive et anarchique, entraînant une dépense inconsidérée dans des ménages dont le budget est pourtant limité. Les boutiques des 'attârîn, les vendeurs d'épices les plus réputés, sont florissantes : leur commerce s'étend aux pays d'alentour, et les cheikhs délivrent de longues ordonnances s'assortissant de prescriptions minutieuses et de conseils de vie qui satisfont le désir de prise en charge globale.

Il n'est pas surprenant, comme Saadia Radi le souligne, que les itinéraires de soins convergent vers les faiseurs de miracles. L'Islam populaire ne manque pas de figures tutélaires auxquelles on peut s'adresser, non plus d'ailleurs que la religion copte. Le tombeau de l'imam Shâfi'î, dans la Cité des morts au Caire, dont Imad Adly se fait le chroniqueur, reçoit les visites de nombreux suppliants désireux de résoudre les problèmes quotidiens. Ils viennent implorer l'intercession d'un saint homme qui fut un grand juriste et le fondateur d'une des quatre écoles de l'Islam. Les nombreux mûlid (fêtes de saints), parmi lesquels les plus fameux sont ceux de Badawî à Tanta (Mayeur-Jaouen, 2004), ou de Sayyida Zaynab au Caire, sont aussi I'occasion d'entrer en relation avec des saints dont l'intervention permet une évasion bienvenue dans le royaume du surnaturel et du miracle. Des lieux également bénis chez les Coptes comme l'arbre de Mariam à al-Matariyya, où la Sainte Famille se serait reposée lors de la fuite en Égypte, la crypte de Sainte Thérèse à Chubrâ où pendent de nombreux ex-voto ( $n a d r$ ) en argent, constituent autant de refuges où tenter de retrouver l'équilibre et la santé perdus, en mobilisant des ressources spirituelles, en harmonie avec les croyances de sa communauté.

L'État qui au temps de Muhammad 'Alî avait assumé la posture d'un État fort, avec un renouveau de puissance lors de l'indépendance nationale et de I'ère nassérienne, adopte en fait une position de repli, comme on l'a vu dans le cas de l'épidémie d'hépatite, vis à vis de ses responsabilités dans le domaine de la santé publique. L'épidémie de sida l'a trouvé également sourd au danger et plus enclin que d'autres pays de la région, comme la Jordanie par exemple, à minimiser le risque et à rejeter la faute sur les moeurs corrompues des étrangers et de quelques compatriotes dévoyés, ainsi que sur les migrants. 
Certes le taux officiel de contamination par le VIH est un des plus bas de la région, surtout si on compare l'Égypte au voisin soudanais. Mais enfin il est probable que le nombre de séropositifs (entre 10 et 50000 ?) est sous-estimé. Le taux annuel d'augmentation de ce nombre serait actuellement de $17 \%$, en dépit de l'existence d'un plan de lutte contre l'épidémie lancé en 1994 (NAP, National AIDS Program). Des mesures comme l'ouverture d'une hot-line destinée à informer ont été prises ainsi que la création d'unités mobiles et fixes de dépistage. Mais le centre de diagnostic anonyme et gratuit d'Alexandrie sous la houlette d'une ONG n'a pas vraiment fait d'émules. Depuis la fin de 2006, il est vrai, les malades ont maintenant accès à une trithérapie gratuite sur indication médicale, mais la délivrance des médicaments s'opère dans un bâtiment du ministère de la Santé au Caire. À intervalles réguliers, des malades d'Alexandrie ou de Sohag sont donc contraints de monter à la capitale. L'absence de respect de la confidentialité fait douter que les malades prennent aisément ce risque d'être repérés et d'affronter la stigmatisation qui les menace. L'État pour le moment assume le coût de ces traitements mais on ne sait pas pour combien de temps encore. II n'a pas négocié d'accords faisant appel à la licence obligatoire, comme il y est autorisé par les accords de Doha, dans la mesure où il ne reconnaît pas l'épidémie comme une urgence l'autorisant à prendre des mesures exceptionnelles pour sauvegarder la santé de ses citoyens, comme passer outre la protection des brevets. II n'est donc pas préparé à traiter une population infectée qui serait plus nombreuse (Khatab, 2007).

Dans ce contexte, le soupçon d'être un réservoir du sida aggrave la situation difficile de la communauté soudanaise réfugiée au Caire et révèle la moralisation, voire la racialisation de l'épidémie. L'épisode violent de décembre 2005, dont la presse internationale s'est fait l'écho, raconté par Minal Giri, illustre la stigmatisation de cette communauté à de multiples titres. L'Égypte a gardé des relations complexes et ambiguës avec un voisin dont elle a partagé I'histoire $^{8}$, dont elle se sent à la fois proche et différente, sans lui être jamais indifférente. Le décalage de prévalence des infections VIH entre Soudanais et Égyptiens sert de détonateur au malaise qui existe devant une immigration qui n'est pas reconnue par les pouvoirs publics et dépasse leurs moyens courants $d^{\prime}$ intervention. Le massacre de réfugiés installés en signe de protestation dans le quartier bourgeois de Mohandisîn a été facilité par des atermoiements des autorités et l'ambiguïté des représentants du Haut-commissariat des Réfugiés au Caire, qui a louvoyé devant une situation explosive et facilité des malentendus sur l'évolution de la situation. L'attitude du gouvernement égyptien, pris entre les remontrances de ses protecteurs américains vis-à-vis de ses infractions aux droits de l'homme, et sa peur d'intervenir dans un domaine aussi sensible que celui des relations sexuelles, aboutit paradoxalement, pour un pays qui n'est pas officiellement fondamentaliste, à maintenir une chape du

8. Sur la genèse de cette ambiguïté, Troutt Powell, 2003. 
secret sur l'épidémie de VIH et ses conditions de transmission. L'État manifeste une incapacité à aborder le problème de la prévention des affections sexuellement transmissibles autrement que de manière moralisatrice et répressive, par la désignation de boucs émissaires et leur bannissement, ou de manière plus feutrée, en laissant un lourd silence s'établir ${ }^{9}$.

Sujet volontiers tabou dans les médias et dans l'éducation scolaire, la sexualité imprègne l'atmosphère de la rue. II n'y a qu'à suivre le regard des policiers en faction devant les bâtiments officiels pour constater la force d'évocation du passage d'une jeune fille devant leurs yeux. Celles-ci, bien que portant le hijâb (le nom signifie protection), se plaignent de harcèlement. Même voilées, certaines d'entre elles ont dû il y a quelques mois affronter en plein centre ville les débordements de jeunes hommes échauffés par la projection d'un film où évoluait une danseuse égyptienne célèbre.

La sexualité est loin pourtant d'être un sujet tabou en terre d'Islam. Les juristes ont été fort diserts sur ce sujet, s'autorisant d'une littérature abondante dès les débuts de I'Islam (Maghen, 2005). La sexualité est aussi un enjeu devant les tribunaux, qui ont à connaître de différents cas de comportements déviant de la norme, et sont amenés à collaborer avec les médecins. Baudouin Dupret, un maître de l'anthropologie juridique, donne quelques échantillons de ces séances de tribunal où les juges ont à trancher entre l'interdit et le tolérable. Le médecin est supposé fournir, de par sa compétence propre, un « contexte » d'interprétation qui pourrait atténuer la portée de telle ou telle condamnation et suggérer des solutions négociées devant certains comportements. En décrivant les processus de fabrication de la preuve médicale apportée au dossier juridique, Baudouin Dupret montre la perte d'autonomie du médical vis-à-vis du juridique et l'alignement des experts sur l'opinion présumée des juges.

La tradition de I'Islam est attachée à une claire division entre les sexes, "Dieu vous a créés mâle et femelle »(Coran), gage de l'ordre social et même cosmique. Pourtant, confronté à l'ambiguïté des comportements sexuels, le médecin peut être amené à reconnaître telle ou telle anomalie anatomique ou physiologique, permettant d'exonérer l'accusé dans certains cas des charges relevées contre lui. Il existe aussi dans la tradition juridique ancienne des procédures amenant à faire jouer toute la gamme de qualifications morales, de l'interdit au toléré et au recommandé (Saunders, 1991). Jusqu'au XIX ${ }^{\mathrm{e}}$ siècle, la tolérance a souvent été réelle vis-à-vis d'une grande variété de conduites sexuelles. En contraste, la médecine semble aujourd'hui participer à une reprise en main de l'ordre moral. Dans les exemples cités par Baudouin Dupret, le médecin désigné comme expert, et recevant comme tel

9. Par contraste, I'Iran des ayatollahs, il est vrai confronté à une épidémie beaucoup plus notable par suite de la conjonction d'un nombre élevé de toxicomanies et de I'afflux des réfugiés afghans, a pris en main la situation de façon plus ouverte et a laissé discuter officiellement un programme national d'information dans les médias. 
une mission précise, tend dans l'énoncé de ses attendus à formuler son avis dans des termes qui tirent son rapport du côté de la moralité plutôt que de la science : par exemple quand il suggère que les constatations anatomiques après examen d'un pédophile supposé peuvent se révéler non concluantes, si ce dernier sait prendre ses précautions : il se situe ainsi clairement sur le versant de l'accusation.

Une des stratégies de l'État égyptien est d'éluder l'analyse des malaises spirituels et psychologiques qui travaillent la société. Les frustrations des citoyens consécutives à la situation intérieure et internationale du pays les amènent à se replier sur l'observance intensive de la religion. Si le fondamentalisme militant a été décapité par l'intense répression qui a suivi les attentats, au cours des dernières années, l'augmentation de la piété populaire est manifeste le vendredi avec l'occupation de la rue par les fidèles ou lors des grandes fêtes religieuses. En même temps, se développe un consumérisme teinté de religiosité et de conformisme et adapté à une société aspirant par ailleurs à la modernité (Haenni, 2005). La vision des familles cairotes, attablées dans les Macdonald, en attendant l'heure de l'iftâr, le repas de rupture du jeûne, en fournit une image caractéristique. Une autre image évocatrice est celle du body-building à l'américaine, introduit dans certains centres de santé islamique, qui fournirait à ses adeptes, de leur propre avis, des dérivatifs bienvenus aux pulsions sexuelles qui les tourmentent, en raison du recul de l'âge du mariage, par suite des difficultés économiques à s'établir.

Vis-à-vis des désordres internes de la société, une des stratégies de l'État est de les attribuer à des maladies mentales et de renvoyer ainsi toute manifestation incongrue à une déviation interprétable dans les termes de la science psychiatrique, comme le montre Ahmed Ragab dans "Madman Walking ». Ce n'est pas la première fois que les psychiatres sont convoqués pour rendre compte de manifestations pathologiques qui pourraient pourtant relever d'autres types d'explications. La criminologie d'inspiration psychiatrique autour de Cesare Lombroso au XIX $x^{\mathrm{e}}$ siècle en donna un bel exemple à propos des anarchistes. À la même époque, la psychiatrie en Égypte débutait au sein de I'hôpital de 'Abbâsiyya sous la houlette du docteur anglais John Warnock en diabolisant la consommation de haschich, alors très répandue dans la population. Warnock assénait que pour plus d'un quart des malades, les problèmes mentaux sont en rapport avec l'abus de haschich. II construisit des batteries de tests et des échelles pour mesurer les déséquilibres associés ${ }^{10}$. Dans le cas des crimes médiatisés évoqués par Ahmed Ragab, la psychiatrisation du désordre social se fait avec la bénédiction du corps médical, invoquée pour détourner l'attention de tout ce qui pourrait faire conclure à un " malaise dans la civilisation » et, à l'occasion d'une révolte individuelle aboutissant à un crime étrange, faire présager des révolutions plus profondes.

10. Journal of Mental Science, 1903, p. 109-110. 
Pour les sciences sociales, la santé apparaît aujourd'hui le terrain d'enquête idéal pour explorer les attentes des populations et leurs désirs de changements, loin des sentiers battus comme à l'écart des terrains trop brûlants de I'action clandestine et des candidats kamikazes. Face aux plaintes des patients, la psychiatrie égyptienne a besoin de réformer profondément ses pratiques et de redéfinir ses valeurs. Doit-elle se borner à être un instrument d'adaptation des sujets à leur environnement, ou peut-elle favoriser une entreprise individuelle d'épanouissement, au péril de non-conformité avec les valeurs affichées de la société ? La société américaine, entre les deux guerres, à propos du duel entre la psychanalyse d'inspiration freudienne et l'école de Karen Horney, s'était posée ces questions. Timothy Mitchell, qui a pratiqué une lecture foucaldienne de l'État mis en place par Muhammad 'Alî (Mitchell, 1988), a inspiré ceux qui attribuent à l'État égyptien des projets soutenus de surveillance panoptique et de conditionnement de la population. Dans cette perspective, la psychiatrie mise en scène par Ahmed Ragab est interpellée dans sa docilité à l'égard de l'État et I'acceptation de sa mission de désamorcer les pulsions de mort des citoyens.

Toutes ces figures de la santé esquissées ici sont sans conteste égyptiennes. Elles ont pourtant un air de famille avec le reste du monde. L'Égypte mère du monde est balayée par les mêmes vents que le reste de la planète (Moulin, 2000). On y retrouve comme ailleurs les propositions de privatisation de la santé et de l'augmentation du rôle des compagnies d'assurances, associée à une segmentation des risques. Le passage d'un État " paresseux et dépensier » à un partenariat public-privé « innovant », pour prendre des expressions consacrées, est préconisé par les grands organismes internationaux et le puissant protecteur américain. La délégation par l'État de ses responsabilités à un citoyen éclairé qui doit se prendre en charge et mettre son comportement en conformité avec les diktats de l'épidémiologie actuelle (alimentation, sexualité, exercice etc.) fait aussi partie du New Age (Porter, 1999). En Égypte comme ailleurs, les gouvernements tendent à incriminer les failles du comportement individuel dit à risque et à rejeter sur les malades la responsabilité de leur pathologie ${ }^{11}$. Les autorités de santé mettent volontiers sur le comportement des ouvriers un certain nombre d'accidents ou de maladies professionnelles. L'opinion publique, de son côté, se soucie de plus en plus de l'environnement, source de pollution atmosphérique, et de l'alimentation qu'elle tient pour « empoisonnée » entre autres par les pesticides. Elle les désigne comme responsables en bloc de la montée des allergies, des affections respiratoires et des cancers.

Parmi ces tendances liées à la " globalisation », il est intéressant d'observer les premiers indices d'un changement à l'égard du tabac dans la société égyptienne. L'Égypte est le paradis des fumeurs : les interdits lancés des États-unis et d'Europe n'ont pas entraîné le bannissement des cigarettes. De plus, la chicha, la pipe à eau, a conquis un espace public sans précédent et atteint tous les

11. Discussion de cette épidémiologie du risque dans Crawford, 1977. 
milieux. La controverse scientifique actuelle sur la nocivité de la chicha (égale à combien de cigarettes ?), en raison de nombreux biais dans les études citées, n'a pas permis pas de conclure (Chaouachi, 2002). Mais d'ores et déjà, signe des temps, même en Égypte, encouragées par l'OMS, des actions de formation sont lancées auprès des jeunes pour les prévenir contre cigarettes et chicha, et faire d'eux le fer de lance d'un changement dans les habitudes urbaines et la sociabilité des cafés.

Comment l'État égyptien, soumis à toutes ces pressions contradictoires, pourrait-il concilier ses ambitions traditionnelles de surveillance et de contrôle avec un désengagement progressif dans le domaine de la santé et une reconnaissance de la société civile et de ses aspirations à une démocratie minimale ? Dans le cas du sida, nous avons vu qu'il choisit en fait l'inaction, et pourtant l'Égypte se situe probablement au tout début de l'épidémie, donc au moment où il serait rentable d'intervenir et possible de stopper, voire d'éradiquer la maladie, en bénéficiant de la longueur d'avance liée à la découverte d'une thérapie relativement efficace et mise en place ailleurs. Les mesures autoritaires de dépistage des étudiants et des résidents étrangers, et de leur expulsion en cas de séropositivité, prises par voie législative depuis 1994, ne suffiront vraisemblablement pas à enrayer la montée inexorable de l'épidémie, malgré un retard marqué vis à vis du reste du monde. Dans un monde où le virus circule inexorablement, c'est l'Égypte qui a tout à perdre, si elle ne prend aucune décision d'envergure, d'une augmentation de nombre de cas sur son territoire. Le pays qui, à défaut d'associations reconnues, commence seulement d'héberger quelques " groupes de parole » et d'entraide risque un jour, comme d'autres pays, d'entrer dans la période moderne de son épidémie. Connaîtrat-il un jour à cette occasion l'émergence d'une nouvelle "biocitoyenneté » dans la "République de la thérapie » ${ }^{12}$ ? Autrement dit, plus généralement, quelles sont les chances pour que les nouveaux problèmes de santé publique entraînent une reconfiguration de la société, et quelle en sera la nature?

\section{VERS UNE RECONFIGURATION DE LA SANTÉ ?}

Si les pressions sont discrètes en ce qui concerne le sida domestique, de la part des organisations internationales qui n'interviennent que très peu dans ce domaine, toute autre est évidemment la situation dès lors que l'épidémie frappant sur le sol de l'Égypte risque en sens inverse de se propager au reste du monde. «Les guerres de la grippe aviaire » illustrent les tensions et les contradictions

12. Nguyen Vim Kinh décrit sous ce nom, dans certains pays d'Afrique, la prise en mains par les associations de malades de la distribution des antirétroviraux et la montée en puissance d'une fraction de la société civile liée à la séropositivité (Nguyen, 2007). 
auxquelles le pays doit faire face. Ici il ne s'agit plus comme pour I'hépatite C d'un fléau national où l'Égypte est renvoyée à son image dans son propre miroir mais de la projection de l'ombre de la pandémie sur la planète. Et il se trouve que pour son malheur l'Égypte, au troisième rang pour le nombre de décès humains, est devenue le point de mire de l'attention internationale.

L'analyse du traitement médiatique de la grippe aviaire de 2005 à 2007 par Matthieu Fintz et Sylla Thierno-Youla est révélatrice une fois de plus du désenchantement des citoyens égyptiens à l'égard du gouvernement. Celui-ci, lent à réagir à l'annonce des premiers cas humains, a choisi secondairement la manière forte, appuyé en cela par les agences internationales agissant au nom du principe de précaution. En médecine vétérinaire, une solution pratique existe en cas de pandémie, l'abattage de masse, jadis appelée méthode de Bang, du nom du Danois qui l'a théorisée. La méthode repose sur une argumentation économique qui fait préférer l'abattage, même quand la vaccination existe. C'est que la vaccination, qui apparaît comme le moyen miracle de préserver les élevages d'une maladie, laisse subsister dans les pays d'importation la suspicion de contamination dans le pays d'origine. Les exportations sont donc peu encouragées voire rendues impossibles, et en tout cas la viande exportable ne peut prétendre à des tarifs intéressants.

En Égypte, après un temps d'hésitation, l'État a pris des mesures brutales de destruction des élevages et d'interdiction de la circulation et de la manipulation des poulets dans les marchés. II a favorisé les élevages industriels, supposés respecter davantage les consignes de surveillance et d'asepsie, et désigné les petits élevages domestiques comme étant à la source des cas humains rapportés de grippe aviaire. Or ces élevages sont une source de précieux revenus additionnels dans les foyers ruraux et même urbains, en particulier pour les femmes dont c'est en quelque sorte la spécialité. Au passage, l'État stigmatise l'arriération des maisonnées vivant au contact étroit des animaux domestiques, critique fréquente chez les voyageurs se déplaçant en Égypte au xix ${ }^{\mathrm{e}}$ siècle et qui figurait déjà dans un rapport de la Compagnie du canal de Suez cité par Céline Frémaux. La guerre de la grippe aviaire, racontée d'après les journaux par M. Fintz et S. Thierno-Youla, est donc plutôt une guérilla où les femmes cachent leurs poussins sous les lits, et où les colombophiles (les magnifiques pigeonniers traditionnels dans les villages sont une fierté de l'Égypte) sont dénoncés comme des agents de propagation de la maladie. Une fois de plus, la santé publique revient, suivant l'expression forte de Paul Farmer, à « blâmer les victimes » (Farmer, 1993).

Le gouvernement a pris des mesures qui lui ont été en grande partie dictées par les organisations internationales, arguant des statistiques inquiétantes des cas humains et de la situation géographique de l'Égypte qui serait placée au carrefour des grands vols migratoires des oiseaux. On pense au poème de Théophile Gautier sur les hirondelles : «L'une dit que dans le vieux Caire/ Il fait bon sur le minaret ». En même temps, le ministre éprouve clairement des difficultés à expliquer sa politique, faisant à la fois face aux pressions internationales, au lobby des élevages industriels de volailles qui forment une partie importante de l'industrie nationale, 
et à son opinion publique. L'annonce de l'avènement probable du poulet congelé laisse planer le soupçon sur l'irruption possible et programmée des multinationales sur le marché égyptien. Outre la perte constituée par le déclin des petits élevages, une telle pratique constituerait aussi un changement de moeurs dans une culture marquée par le souci de l'abattage rituel islamique et d'un certain mode de consommation de la viande. Ailleurs en Inde, par exemple, l'introduction du fast food a pu déclencher de véritables émeutes locales, parce qu'il paraissait contraire à la culture et à la gastronomie, et surtout parce qu'il symbolisait l'intrusion des multinationales alimentaires (Dalrymple, 1998).

Si la gestion de la grippe aviaire suscite surtout la colère des petits éleveurs et des paysans et la verve des journalistes trouvant dans cet épisode des armes métaphoriques pour décrire les turpitudes de l'État policier, en revanche la réforme de la santé concerne tout le monde. Il est d'autant plus étonnant, comme le montre Françoise Clément, que les plans de réforme de l'assurance maladie, en discussion depuis plusieurs années, ne soient pas connus dans le détail par le grand public. L'assurance maladie, mieux nommée en Égypte I'assurance santé, a été en effet dénoncée comme à la fois financièrement déficitaire et responsable de l'état insatisfaisant du système de santé. Les projets de réforme se sont succédé sans être connus dans le détail, puisqu'ils ne sont pas publiés exhaustivement avant d'être discutés devant le Parlement.

Françoise Clément nous initie au détail des projets successifs de réforme depuis I'an 200013. Fortement influencées par la Banque mondiale et les bailleurs de fonds étrangers, ces réformes sont marquées au coin de l'évolution libérale qui amène à prôner dans tous les domaines le retour aux mécanismes du marché et à la compétition comme la panacée. Le gouvernement égyptien doit donc résoudre le grand écart. II doit satisfaire les aspirations de la population en améliorant la couverture du risque maladie et en l'étendant aux nombreuses catégories exclues, soit près de la moitié de la population, puisque la couverture universelle apparaît comme un droit légitime des citoyens des temps modernes. Il doit en même temps améliorer la qualité des soins, dont les journaux dénoncent l'insuffisance chronique. La séparation du financement et de la gestion des soins est proposée comme le remède miracle, en supposant que la sous-traitance voire la privatisation de beaucoup d'activités médicales améliorera l'efficacité et la rentabilité de la prestation des soins. Tout cela ne risque-t-il pas d'être wishful thinking, rester un voeu pieux? La privatisation et la compétition permettront-elles vraiment de rendre les temps de travail plus effectifs et les soins mieux dispensés ? De toute façon, une augmentation substantielle du budget santé est nécessaire pour répondre à toutes ces attentes ; les projets de réforme présentent donc l'accroissement de la participation des usagers au coût des prestations et la segmentation des risques comme la clé de cette augmentation de ressources. L'État ne garantirait plus que certains

13. Voir aussi Fintz, 2006. 
programmes comme les soins de base, toujours à redéfinir : la santé maternelle et infantile, les vaccinations préventives des grandes endémies...

Mais même cette définition du " paquet minimum garanti » est sujette à caution. Ce raisonnement valait quand la technologie avait un coût limité et qu'on s'en tenait à de bons vieux vaccins dont les brevets étaient éteints depuis longtemps, comme le vaccin antitétanique ou même le vaccin antipolio (Moulin, 2007). Prenons un exemple. Que se passera-t-il quand, comme il est prévu, la vaccination préventive du cancer de l'utérus sera implantée en Égypte, grâce à la fondation GAVI (Global Alliance for Vaccine Initiative). Il s'agit d'un tout nouveau vaccin récemment promu par l'industrie pharmaceutique à destination des fillettes prépubères, à la suite de travaux prouvant le lien entre une affection virale sexuellement transmissible et le cancer du col de l'utérus, au deuxième rang de la mortalité par cancer chez les femmes. Dans un premier temps, ce vaccin sera procuré gratuitement, à charge pourtant pour le gouvernement de I'appliquer en campagne de masse. Cet exemple illustre comment les décisions de l'État en matière de santé publique peuvent être influencées par une organisation internationale dont les buts sont certes humanitaires mais qui effectue ses propres choix dans les priorités de santé publique, qui de toute façon n'ont pas été démocratiquement discutés (Muraskin, à paraître). D’autre part, comment cette vaccination sera-t-elle expliquée dans son principe? Comment la population comprendra-t-elle une mesure qui revient à vacciner les fillettes avant la puberté contre l'éventualité d'une MST ? Le gouvernement, embarrassé et peu soucieux d'entrer dans un débat sur le risque de MST projeté dans I'avenir d'une fillette égyptienne, préfèrera probablement s'abstenir d'explications sur la science en train de se faire. Il pourra dès lors légitimement être interpellé sur le champ libre laissé aux industries pharmaceutiques promouvant ce nouveau vaccin. À moins que le caractère trop technique du débat ne le dérobe à la place publique.

À la lecture de ce dossier, peut-être le lecteur trouvera-t-il le panorama trop pessimiste. L'équipe qui a coordonné ce numéro est responsable sinon du contenu des articles, du moins de l'orientation donnée à leur ensemble. Elle voudrait, avant de laisser la parole aux différents contributeurs, souligner certains points. D'une part, il s'agit d'un numéro où la parole est aux sciences sociales, qui par définition ont une posture critique et non patrimoniale des biens de société. La santé, le bien reconnu à l'unanimité comme un des plus précieux, invoqué constamment sous la sauvegarde de Dieu dans la conversation de tous les jours, est aussi un bien fragile, et l'inégalité des citoyens en matière de santé est probablement un des indicateurs les plus précieux à retenir pour porter un jugement sur une société. Certains économistes désignent les sociétés où les inégalités de santé sont particulièrement marquées comme des sociétés globalement unhealthy, malsaines ou malades, un jugement de valeur qui va loin (Wilkinson, 1992). Ces images ont aussi cours en Égypte où, des Frères musulmans aux avocats du despotisme éclairé d'un raïs, chaque parti mobilise la figure d'une société malade, décadente et corrompue pour justifier son emprise sur un peuple jugé infantile. 
Pour illustrer la couverture, nous avons préféré tourner le dos à la maladie et choisi le portrait d'un jeune étudiant d'Al-Azhar qui se penche avec enthousiasme sur un microscope pour déchiffrer les mystères de la vie. Ce n'est bien sûr qu'un instantané, la trace dans les années cinquante d'une alliance entre tradition et modernité, science et religion, par définition instable. Chaque génération réinvente et négocie de nouveaux itinéraires intellectuels et thérapeutiques. Le composé corps esprit demeure ombres et lumières, transparent et mystérieux, au carrefour de mondes qui se pénètrent sans s'opposer nécessairement, croyances et connaissances, certitudes et doutes. Les outils de la médecine, bistouris, drogues, seringues sont à la fois attrayants et effrayants, magiques et banals. Ainsi le stéthoscope incarne la modernité tandis que le sibha, le chapelet qu'on égrène pour passer le temps ou invoquer Dieu dont vient toute guérison, reflète la tradition. Mais l'auscultation est détrônée par les méthodes d'enregistrement sophistiquées et le stéthoscope tend à être relégué au rang de gadget de la profession, tandis que la médecine prophétique retrouve de nouveaux adeptes. Vert et rouge, les couleurs choisies pour la couverture illustrent le double registre la peur et de l'espérance de l'Egypte contemporaine, au seuil d'une possible reconfiguration de la santé.

Pour porter un jugement synthétique sur la santé de l'Égypte, il est clair qu'il faudrait une plus grande variété de sondages et une information plus exhaustive que celle que nous présentons ici. C'est pour cela que nous avons choisi le titre Figures de la santé en Égypte, pour ces aperçus rapides et nous l'espérons pénétrants, formulés avec une certaine vivacité, voire véhémence, à l'image de la parole égyptienne dans la vie de tous les jours. La santé n'est pas une abstraction, elle est ici saisie à travers les figures concrètes du médecin, du saint, de l'homme d'État, de l'usager de soins, dans des lieux également concrets qui sont l'hôpital, le sanctuaire, le tribunal, le café, la ferme. Mais cette succession suggère aussi une figure globale de la société égyptienne, son vécu, ses attentes et ses déceptions, marquée par l'humour avec lequel les gens savent toujours se saisir du temps qui vient, de la minute qui passe, en considérant que, qui sait? comme le dit une nukta, une blague locale, aujourd'hui est probablement meilleur que demain.

Si la santé publique est d'ancienne venue en Égypte, les politiques de santé ont toujours été décidées d'en haut pour s'appliquer en bas, disjointes de la citoyenneté. Cette verticalité pourrait expliquer que les malades privilégient des stratégies individuelles d'accès aux soins et renoncent à exiger collectivement plus d'efficacité et de meilleurs soins. On a vu que les associations de malades, même dans le cas de maladies aussi répandues que l'hépatite $C$, n'existent pas. Ce qui en tient lieu est soit des réseaux d'entraide soit des associations patronnées par des médecins. Tout se passe finalement comme si l'Égypte, ayant pourtant très tôt implanté le système biomédical sur son sol, ne l'avait jamais réellement mis à la disposition des citoyens. À moyen terme, il paraît cependant probable que des exigences plus grandes se manifestent en matière de santé. La grippe aviaire, si le virus connaissait en Égypte une mutation entraînant la 
transmission interhumaine, pourrait servir de détonateur, dans la mesure où la catastrophe révèlerait crûment les insuffisances du système de santé. Sans se prononcer sur la réalité de ce risque, notons seulement qu'il est pris au sérieux par de nombreux acteurs sociaux. L'évolution des politiques de santé sera dans les années qui viennent un test des relations entre gouvernants et gouvernés et des chances pour le pays d'améliorer la santé de ses membres. L'hépatite, ou la menace de la grippe aviaire, s'avèreront-elles finalement réformatrices ?

Ibn Ridwân concluait son traité médical :

Les maux des Égyptiens sont simples et sans complications. Leur traitement est aisé et leurs conséquences peut-être pas si graves que cela, en ce qu'ils ne les placent pas dans de dangereuses situations de détresse. L'Égypte entraîne peu de discordes et de guerres en raison de leur acceptation de ceux qui les gouvernent et de la faiblesse de la résistance que les habitants leur opposent. La vie en Égypte est préférable et les bénéfices à ceux qui y vivent sont nombreux. (Ibn Ridwân, dans Dols, p. 148-9)

Serait-il vrai que, en dépit des vents contraires qui balayent le pays du Khamâsîn, la santé n'entraîne pas une mobilisation plus intense parce que ses habitants épris de paix s'adaptent avec constance à leurs maux ou faut-il envisager une reconfiguration différente dans un proche avenir ?

\section{RÉFÉRENCES BIBLIOGRAPHIQUES}

'ABBÂs R (dir.), 2000, Islâm am tahdîth? Misr fî 'asr Muhammad 'Alî (Réforme ou modernisation? L'Égypte à l'époque de Muhammad 'Alî), Le Caire.

Al Zanaty F et Way Ann A, in National Population Council Egypt (NPC)/ Demographic and Health Surveys, 2001, Egypt Demographic and Health Survey 2000, Cairo 2001.

Ayrout H, 1938, Moeurs et coutumes des fellahs d'Égypte, Paris, Payot.

Beatty C, 1957, Ferdinand de Lesseps, Biographie, Paris, Del Duca.

Blackman W.S., 1948, Les fellahs de la Haute Égypte, Paris, Payot.

Boggatz T et Dasson T, 2005, "Ageing, care dependency and care for older people, in Egypt, a review of the literature », Journal of Clinical Nursing, 14, 8B, p. 56-63.

CAMPION-VINCENT Véronique, 1996, La greffe, les rumeurs et les médias. Les récits de vol d'organes, Paris, Maison des sciences de I'Homme.

ChaOuachi K, 2002, Le monde du narguilé, Paris, Maisonneuve et Larose.

Chiffoleau S, 1997, Médecine et médecins en Égypte, Paris, L'Harmattan.

Clot A.B, 1840, Aperçu général sur l'Égypte, t II, Paris, Fortin et Masson.

Costet-Tardieu F, 2005, Un réformiste à l'université d'al-Azhar. OEuvre et pensée de Mustafâ al-Marâghîi (1881-1945), Paris/Le Caire, Karthala/Cedej.

CourbageY, 1994, «La politique démographique en Égypte et son évaluation ? Que nous apprennent ces enquêtes récentes ? », Population, 4-5, p. 1041-1056. 
Crawford R., 1977, "You are responsible for your health : the ideology and politics of victim blaming », International Journal of Health Services, 7 (4), p. 663-668.

Dalrymple W, The Age of Kali, Indian Travels and Encounters, Harper Collins, London 1998, pp. 158-173.

Dots M, 1972, The Black Death in the Middle East, Princeton, Princeton University Press.

- 1984, Medieval Islamic Medicine, Ibn Ridwan's Treatise on the prevention of bodily ills in Egypt, Berkeley, University of California Press.

- 1987, "The origins of the Islamic hospitals. Myth and reality », Bulletin of the History of Medicine, 61, p. 367-390.

ERCKMANn-Chatrian, 1963, "Un chef de chantier dans l'isthme de Suez » dans Contes et romans nationaux et populaires, Paris, réédition Pauvert, p. 267-410.

Fahmi K, 1997, All the Pasha's Men : Mehmed Ali, his army and the making of modern Egypt, Cambridge, Cambridge University Press.

FARLEY J, 1991, Bilharzia : a History of Imperial Tropical medicine, Cambridge, Cambridge University Press.

Farmer Paul, 1993, Haiti and the Geography of Blame, Berkeley, California University Press.

Fathy H, 2004, Architecture for the Poor. An experiment in Rural Egypt, [1 1 ère édition 1969], Le Caire, the American University in Cairo.

Fintz Mathieu, 2006, "A Reform for the Poor without them? » dans L'Égypte dans l'année 2005, Kohstall F (dir.), Le Caire, Cedej.

Frank C, Mohamed M.K. et al., 2000, The role of parenteral shistosomal therapy in the spread of hepatitis C virus in Egypt, Lancet, 355, 9207, p. 887-91.

Gallagher N., 1990, Egypt's Other Wars : Epidemics and the Politics of Public Health, New York, Syracuse University Press.

Goubert J-P (Goubert dir.) 1982, La médicalisation de la société française (1770-1830), Waterloo (Ont.), Historical Reflections Press.

HaENNi P, 2005, L'Islam de marché. L'autre révolution conservatrice, Paris, Le Seuil.

Iluıch I, 1975, Némésis Médicale, Paris, Le Seuil.

Jaffré Y et SARDAN (de) O, 2004, La médecine inhospitalière, Paris, PUF.

Кнатав H, 2007, Report on a study of women living with HIV in Egypt, Le Caire, Egyptian Society for Population Studies and Reproductive Health.

LÉONARD J, 1981, La médecine entre les pouvoirs et les savoirs, Paris, Aubier.

MAGHEN Ze'ev, 2005, The Virtues of the Flesh, Passion and purity in early Islamic jurisprudence, Leyde, Brill.

Mayeur-Jaouen Catherine, 2004, Histoire d'un pèlerinage légendaire en Islam. Le mouled de Tanta du 13e siècle à nos jours, Paris, Aubier.

MikHAIL Alan, 2007, "Counting men, measuring canals, drawing maps : the science of irrigation in Ottoman Egypt », Communication du 29 mai 2007 au Cedej. 
Mitchell T, 1988, Colonizing Egypt, Cambridge, Cambridge University Press. - 2002, Rule of Experts, Egypt, Techno-politics, Modernity, Berkeley, University of California Press.

Moriconi-Ebrard F et Bayoumi H, 2005, One Century Census, Egypt 18821996, Le Caire, Cedej-CAPMAS.

Moulin A.M., 1991, "Bioéthique en terres d'Islam » dans Bio-éthique et cultures, Paris, UNESCO/Vrin, p. 85-96.

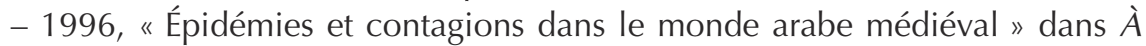
I'ombre d'Avicenne, La médecine au temps des califes, Paris, Institut du Monde arabe, p. 247-260.

- 2000, «Les sociétés au siècle de la santé », Médecine/Sciences, 11, p. 11851191.

- 2004, "Ordre et désordre dans le champ de la santé » dans F-X SCHWEYER, S. Pennec, G. Cresson et F. Bouchayer (dirs), Normes et Valeurs dans le champ de la santé, Rennes, Éditions ENSP.

- 2007, "Les vaccins, I'État moderne et les sociétés », Médecine/Sciences, 21, p. 1-7.

MURASKIN W, à paraître, Revolution in international public health. The origins and development of the Bill and Melinda Gates Children Vaccine Program and the Global Alliance for Vaccines and Immunization, Rochester University Press.

NGuyen V-K, 2007, The Republic of Therapy : biopolitics before and after HIV in French West Africa, Durham, Duke University Press.

OmRAn A.R., 1971, "The epidemiological transition. A theory of the epidemiology of populations changes », Millbank Memorial Fund Quarterly, 29, p. 509-538.

Porter D, 1999, Health, Civilization and the State, Londres, Routledge.

RADI S, 2006, "Débats de presse, scandale et mise en place d'une politique de prévention. À propos de I'hépatite C », Revue d'Epidémiologie et de Santé publique, 54, p. 1545-1552.

Sardar Z, 1984, The Touch of Midas, Science, Values and Environment in Islam, Manchester, Manchester University Press.

Saunders P, 1991, "Hermaphrodites » dans N. R. Keddie et B Baron (dirs), Women in Middle East History, Shifting boundaries in Sex and Gender, Yale, Yale University Press, p. 74-121.

Troutt Powell E.M., 2003, A Different Shade of Colonialism. Egypt, Great Britain and the mastery of Sudan, Berkeley, University of California Press.

TurC A, s d, "Une ethnographie de la "santé au quotidien"en Égypte: la médecine moderne entre les affres de la bureaucratie et l'influence de la religion » dans A.M. Moulin (dir.), Le labyrinthe du corps. Islam et modernité médicale, à paraître.

WiLkInson R.G., 1992, Unhealthy Societies, The Afflictions of Unequality, Londres, Routledge. 